\title{
Implantación de semi-presencialidad en una asignatura optativa de la licenciatura en Ciencias y Técnicas Estadísticas.
}

\author{
Erik Cobo; José A. González \\ Erik.Cobo@upc.es; Jose.A. Gonzalez@upc.es \\ Departamento de Estadística e Investigación \\ Operativa, Universidad Politécnica de Cataluña \\ $(U P C)$, Barcelona
}

\section{Introducción}

Aplicaciones de la Estadística en las Ciencias de la Salud es una asignatura de 7.5 créditos que se ofrece bienalmente a los estudiantes de la Licenciatura de Ciencias y Técnicas Estadística, titulación de segundo ciclo de la Facultad de Matemáticas y Estadística (FME). Dos tercios de los alumnos son diplomados en estadística por una de las tres universidades catalanas (UAB, UB y UPC) que ofrecen estos estudios; y una cuarta parte son, o bien recién licenciados en matemáticas, o bien estudiantes que cursan simultáneamente la doble titulación en Matemáticas y en Estadística que oferta la FME. Como el horario de clase es por la tarde, la mayor parte de los estudiantes pueden compaginar sus estudios con su trabajo como estadísticos, generalmente como becarios en centros de investigación biomédica.

Se trata por tanto de estudiantes maduros, con altos niveles de motivación. Por el perfil de la licenciatura, tienen una sólida formación teórica en estadística matemática, investigación operativa, fundamentos matemáticos especialmente en álgebra y cálculo-, informática, bases de datos, modelado estadístico y diseño de experimentos. Por su implicación profesional desean conocer técnicas estadísticas concretas que se aplican en las ciencias de la salud. Así, se matriculan en la asignatura con el deseo de practicar, por ejemplo, sus conocimientos de diseño de experimentos en el análisis de un ensayo clínico; y los de modelado multivariante en el análisis de diseños observacionales. Responder a esta demanda podría representar el riesgo de convertir la asignatura en un mero recetario de procedimientos estadísticos que no incidiría en los aspectos formativos que reclama la declaración de Bolonia (Ministros de Educación europeos, 1999) y que, dado el rápido progreso de la estadística, implicaría formar alumnos en técnicas que, en mayor o menor grado, tienen fecha de caducidad temprana.

Por otro lado, ofertar la asignatura en modo semi-presencial podría representar ventajas para aquellos estudiantes con mayores restricciones por su ejercicio profesional. Por estas razones, y de acuerdo con la dirección de estudios de la FME, se procedió a rediseñar la asignatura para ofertarla a los alumnos durante el segundo cuatrimestre del curso 2004-2005.

En el presente artículo se pretende hacer una valoración de la experiencia. En el siguiente apartado se comentan los objetivos docentes; en el tercer apartado, la metodología y estructura de la asignatura; en el cuarto, los resultados obtenidos; y en el apartado final, se ofrecen las conclusiones y consideraciones a tener en cuenta en futuras ediciones.

\section{Objetivos Docentes}

¿Qué cualidades deben diferenciar a un estadístico profesional? Se cuenta que, en un viaje a un congreso, coincidieron en el tren dos estadísticos y dos investigadores. Los primeros viajaban con un solo billete que, escondidos en el lavabo, pasaron al revisor por debajo de la puerta. A la vuelta del congreso, los estadísticos viajaron sin billete, pues usaron el que los investigadores aplicados les pasaron por debajo de la puerta antes de ser multados. Esta historia termina en moraleja: "nunca use un procedimiento estadístico si no domina bien su técnica". En efecto, los paquetes informáticos de estadística han facilitado su uso y contribuido a su difusión, pero también han puesto a disposición de mentes imprudentes, herramientas sofisticadas que son usadas de 
forma inadecuada (Von Elm. y Egger, 2004), perpetuándose los errores dado el proceso de emulación por el que los investigadores suelen aprender el método científico (Altman y Goodman, 1994).

Para romper este círculo, se precisan estadísticos capaces de interactuar con investigadores de alto nivel en su disciplina pero con vicios adquiridos en su conocimiento metodológico. En esta línea, alumnos de ediciones previas de esta asignatura fueron incluidos por los profesores como revisores estadísticos en la revista semanal Medicina Clínica (factor de impacto > 1) y participaron en un diseño experimental para valorar el efecto, en la calidad del informe final, de añadir un revisor estadístico a los revisores clínicos (Arnau, Cobo et al. 2003), en el que se constató una gran heterogeneidad en las opiniones y sugerencias de los estadísticos a los autores y a los editores. Esta experiencia fue tomada como base para formar estadísticos que fueran capaces de integrarse en un equipo de investigación multidisciplinar, aportando soluciones en todas las fases del proyecto de investigación, desde el diseño hasta el informe. Se observó que las habilidades requeridas coincidían en gran manera con las reseñadas en la convergencia al nuevo espacio europeo de educación superior (MEC, 1999) y decidimos diseñar una asignatura que facilitara el desarrollo en los alumnos de las capacidades recogidas en el cuadro 1, seleccionadas de la documentación de la adaptación al Espacio Europeo de Educación Superior de la Facultad de Matemáticas y Estadística de la UPC (Heredia, 2005).

\begin{tabular}{|l|}
\hline \multicolumn{1}{|c|}{ Cuadro 1: competencias que se pretenden desarrollar } \\
\hline Competencias específicas profesionales \\
\hline Capacidad para resolver las necesidades de análisis de la información de las \\
organizaciones, identificando las fuentes de incertidumbre y variabilidad. \\
Capacidad para resolver los problemas de decisión de las organizaciones \\
integrando los resultados de los análisis estadísticos. \\
Capacidad de diseñar, redactar y presentar informes estadísticos. \\
Respeto a la norma legal y deontológica en su ejercicio profesional. \\
\hline Competencias genéricas \\
\hline Capacidad para expresarse cuantitativamente \\
Capacidad de análisis y síntesis. \\
Razonamiento crítico. \\
Comunicación oral y escrita. \\
Uso profesional de la lengua inglesa. \\
Aprendizaje autónomo. \\
Capacidad de trabajo en equipos multidisciplinares. \\
Motivación por el trabajo bien hecho. \\
Iniciativa, espíritu emprendedor y creatividad. \\
\hline
\end{tabular}

La estadística, como toda ciencia, tiene diferentes escuelas. Desgraciadamente, al exportar la discusión se ha facilitado la sensación de que la estadística podría no tener las "cosas claras". Así, es conocido el consejobroma de poner los estadísticos en número par en un comité para anular su influencia. Por ejemplo, las dos opciones alternativas de reflejar el nivel de significación exacto (sea $\mathrm{P}=0$ ’0023) o decir tan sólo el nivel del riesgo de primera especie (sea $\mathrm{a}=0$ '05) responden a dos perspectivas epistemiológicas claramente diferenciadas (Ver Berger, 2003, y Hubbard y Bayarri, 2003): la búsqueda de R.A Fisher de argumentos probabilísticos en contra de una hipótesis que se desea rechazar - que encaja con el falsacionismo de K Popper (1967) - y el planteamiento de Neyman-Pearson para optimizar la decisión entre dos alternativas. El científico no capta la distinción entre "¿qué sé?” y “¿qué hago?", ya que emplea rutinariamente la estadística como motor en su adquisición de conocimiento, pero la interpreta como criterio de decisión. Por esta razón, progresivamente se ha ido sugeriendo el empleo de técnicas estadísticas que no induzcan a confusión, como podrían ser los intervalos de confianza. Asimismo, se han desarrollado guías metodológicas para el informe de resultados con el objetivo de facilitar al máximo su interpretación, independientemente del nivel metodológico del lector, y contribuir así 
tanto a la difusión de la ciencia como al uso correcto de los métodos estadísticos. Fruto de estas iniciativas, aparecieron en 1998 los CONsolidated Standards of Reporting Trials (CONSORT, de Moher, 1998) que proponen un modelo para el informe de los resultados de ensayos clínicos en revistas biomédicas, y al que han seguido diversas guías para otros tipos de diseños (STARD, de Bossuyt et al, 2003; STROBE, ver Fernández, 2005; QUOROM, de Moher, 1999, etc.). Como asignatura optativa situada al final del ciclo formativo, nos planteamos incluir en los objetivos académicos (cuadro 2) el conocimiento de estas guías.

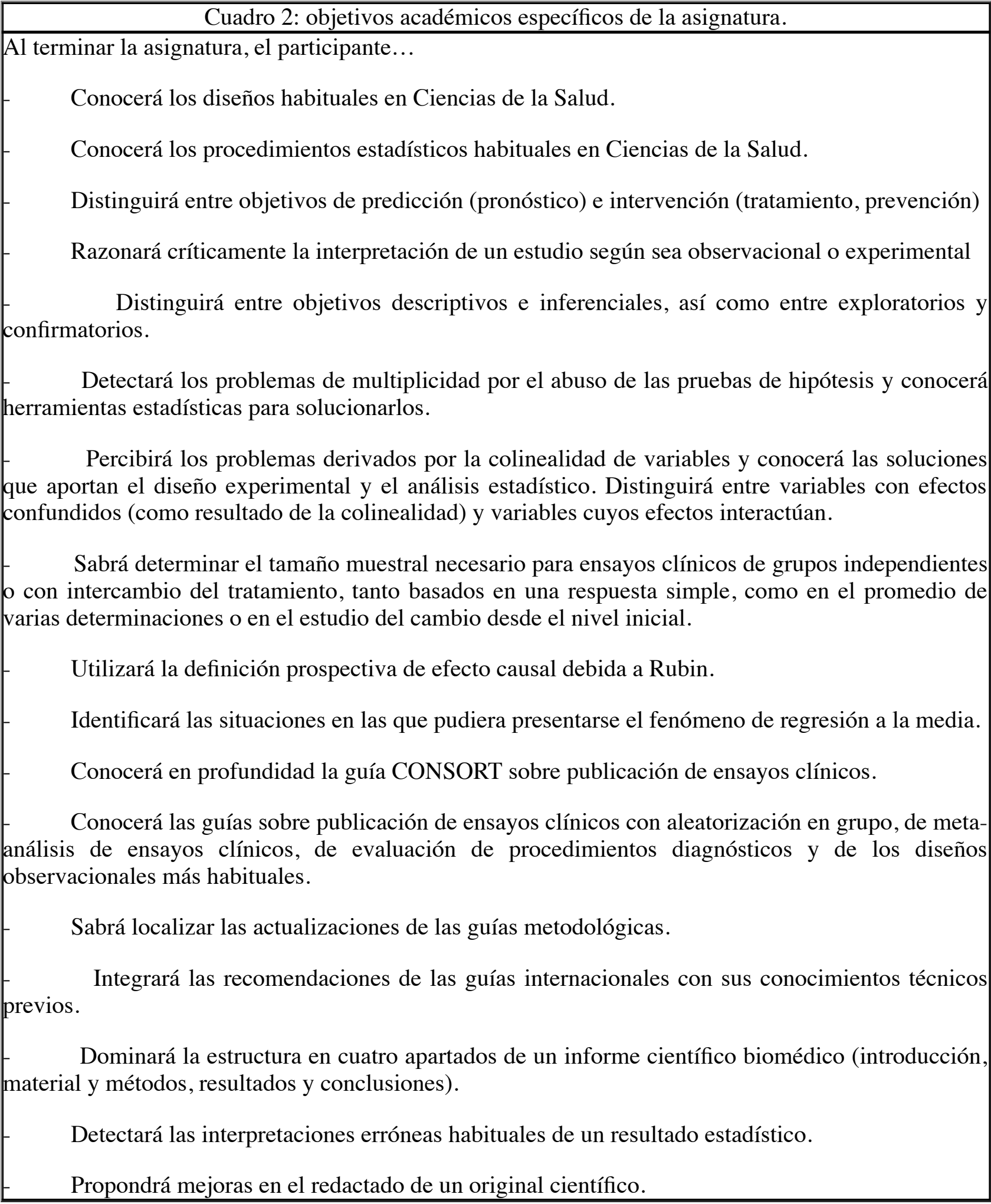

\section{Metodología docente y estructura de la asignatura.}

Para conseguir todos estos objetivos, se decidió emplear los métodos didácticos que se describen a continuación.

- Campus virtual ATENEA: desarrollado en nuestra Universidad y adoptado, entre otros centros, por la FME. Permite colgar documentación tanto por el profesorado como por el alumnado, mantener foros de 
discusión, seguir la agenda docente, publicar de forma anónima la evaluación del alumno, realizar conexiones docentes y, en general, todas las funciones habituales de las plataformas docentes. Sin su existencia y fiabilidad, este planteamiento de la asignatura no habría sido posible.

- Trabajo cooperativo: los alumnos preparan, en grupos de tres, el apartado de una guía metodológica concreta (por ejemplo, la CONSORT) y lo presentan oralmente en clase a sus compañeros. Reciben retroalimentación oral del profesorado y escrita de sus compañeros, que deben evaluarlos de acuerdo con el nivel mostrado en 1) dominio de los conocimientos; 2) estilo de la presentación; 3) claridad en la exposición; y 4) concisión.

- Prácticas: mediante el trabajo en grupo, los alumnos aplican una guía metodológica, como la CONSORT, a la lectura crítica de estudio empírico publicado en una revista internacional. La presentan por escrito y reciben retroalimentación del profesorado.

- Evaluación: a nivel individual, con la ayuda de libros y usando las guías metodológicas, el alumno debe detectar errores en artículos de publicaciones internacionales y ofrecer sugerencias para mejorar el diseño, el análisis o el informe. Consiste en tres evaluaciones formativas o parciales y una evaluación final que, a modo de ejemplo, se presenta en el cuadro 3. La evaluación formativa se obtiene de acuerdo con las ponderaciones que figuran en el cuadro 4. Aquellos alumnos que hayan superado el 6' 5 de nota pueden optar por no presentarse al examen final. Se devuelven corregidas individualmente de acuerdo con el modelo que se les entrega.

Resolución de ejercicios con la aplicación informática e-status que genera datos diferentes para cada alumno y ofrece retroalimentación inmediata sobre el nivel de acierto y se presenta en otro artículo de este mismo número (González et al, 2006).

Participación: a partir de una pregunta concreta sobre un tema controvertido, se pide a los alumnos que opinen en el foro de discusión. Por ejemplo, la misma semana en que el alumno aprende que el ensayo clínico aleatorizado es obligatorio antes de aprobar el uso de una nueva intervención médica, se les pregunta qué premisas lo hacen innecesario para valorar el efecto del paracaídas en saltos desde mil metros de altura (Gordon et al, 2003).

\begin{tabular}{|l|}
\hline \multicolumn{1}{|c|}{ Cuadro 3: evaluación final. } \\
\hline $\begin{array}{l}\text { Por favor, lea atentamente el original adjunto. [Puede realizar en voz alta } \\
\text { cualquier consulta terminológica.] }\end{array}$ \\
1) ¿Cuál es, a su entender, la(s) forma(s) en la(s) que más podría ser mejorado el \\
análisis estadístico del presente original? (4 puntos) \\
2) Como cualquier estudio de intervención sobre hábitos de vida, el paciente \\
conoce el grupo al que ha sido asignado. ¿Qué implicaciones tiene? ¿Cómo \\
deben ser el diseño y el análisis para mantener la máxima calidad? (4 puntos) \\
4) Por favor, realice otras sugerencias para mejorar el original. (2 puntos)
\end{tabular}

Todas las exposiciones orales de los profesores fueron sustituidas por presentaciones de los alumnos, limitándose los profesores a dar retroalimentación. Para fomentar la autoestima de los primeros, necesaria para desarrollar los objetivos docentes, se decidió que los comentarios de los segundos fueran tan positivos como permitieran las intervenciones de los alumnos y más que resaltar los puntos débiles no detectados, se centraría la discusión en los puntos débiles que los alumnos sí habían descubierto.

El cuadro 4 presenta la estructura final del curso, que no coincidió con la planificada por tres razones: 1) se había infravalorado la carga de trabajo; 2) se adaptaron las entregas al ritmo de otras asignaturas; y 3) la primera presentación tuvo que repetirse debido a su bajo nivel.

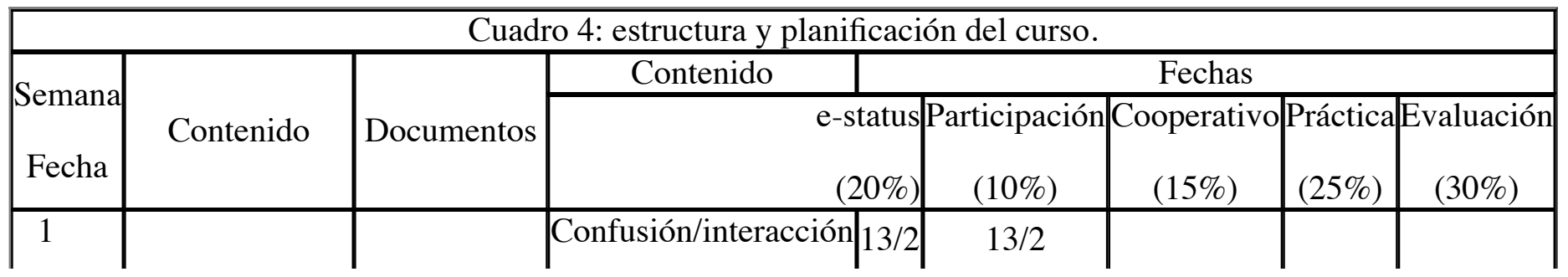




\begin{tabular}{|c|c|c|c|c|c|c|c|c|}
\hline $7 / 2$ & \multirow{4}{*}{$\begin{array}{c}\text { Ensayo Clínico } \\
\text { Aleatorizado }\end{array}$} & \multirow{4}{*}{ CONSORT } & & & & \multirow[b]{2}{*}{$21 / 2$} & & \\
\hline $\begin{array}{l}2 \\
14 / 2\end{array}$ & & & Tamaño muestral & $20 / 2$ & $20 / 2$ & & & \\
\hline $\begin{array}{l}3 \\
21 / 2\end{array}$ & & & Multiplicidad & $27 / 2$ & $27 / 2$ & \multirow[b]{2}{*}{$28 / 2$} & & \\
\hline $\begin{array}{c}4 \\
28 / 2 \\
\end{array}$ & & & \multicolumn{2}{|c|}{$N O H A Y$} & NOHAY & & & \\
\hline $\begin{array}{l}5 \\
7 / 3\end{array}$ & \multirow{2}{*}{ ECA en grupo } & \multirow{2}{*}{$\begin{array}{c}\text { Cluster } \\
\text { CONSORT }\end{array}$} & \multicolumn{2}{|c|}{ NOHAY } & $13 / 3$ & & & \\
\hline $\begin{array}{c}6 \\
14 / 3 \\
\end{array}$ & & & \multicolumn{2}{|c|}{ NOHAY } & NO HAY & NOHAY & $14 / 3$ & $14 / 3$ \\
\hline \multicolumn{9}{|l|}{$21 / 3$} \\
\hline $\begin{array}{c}7 \\
28 / 3 \\
\end{array}$ & \multirow{5}{*}{ Meta-análisis } & \multirow{5}{*}{$\begin{array}{l}\text { QUOROM } \\
\text { COCHRANE }\end{array}$} & Meta-análisis & $10 / 4$ & NOHAY & & & \\
\hline $\begin{array}{l}8 \\
4 / 4\end{array}$ & & & \multicolumn{2}{|c|}{$N O H A Y$} & $10 / 4$ & $11 / 4$ & & \\
\hline $\begin{array}{l}9 \\
11 / 4\end{array}$ & & & \multicolumn{2}{|c|}{$N O H A Y$} & $17 / 4$ & & & \\
\hline $\begin{array}{c}10 \\
18 / 4 \\
\end{array}$ & & & \multicolumn{2}{|c|}{ NO HAY } & NOHAY & & & \\
\hline $\begin{array}{c}11 \\
25 / 4\end{array}$ & & & \multicolumn{2}{|c|}{ NOHAY } & NOHAY & $18 / 4$ & $18 / 4$ & $25 / 4$ \\
\hline $\begin{array}{l}12 \\
2 / 5\end{array}$ & \multirow{3}{*}{$\begin{array}{c}\text { Intervención no } \\
\text { aleatorizado y } \\
\text { Observacionales }\end{array}$} & \multirow{3}{*}{$\begin{array}{l}\text { TREND } \\
\text { STROBE }\end{array}$} & Regresión media & $1 / 5$ & NOHAY & & & \\
\hline $\begin{array}{l}13 \\
9 / 5 \\
\end{array}$ & & & \multicolumn{2}{|c|}{ NO HAY } & NO HAY & $9 / 5$ & & \\
\hline $\begin{array}{c}14 \\
16 / 5\end{array}$ & & & \multicolumn{2}{|c|}{ NO $H A Y$} & $15 / 5$ & NO HAY & $18 / 5$ & $18 / 5$ \\
\hline
\end{tabular}

\section{Resultados}

Se inscribieron en esta asignatura optativa 22 alumnos, y uno de ellos, profesor de secundaria, la abandono a los quince días argumentando que no podía asumir la carga de trabajo prevista durante el curso y que su estilo era el de estudiar a solas para preparar el examen final. Los 21 alumnos restantes superaron la asignatura con éxito; al examen final se presentaron 5 alumnos, 3 para mejorar nota y 2 porque no alcanzaban el 6' 5 requerido para liberar.

\begin{tabular}{|c|c|c|c|}
\hline \multicolumn{4}{|c|}{ Cuadro 5: evaluación cuantitativa del curso. } \\
\hline & Escala de 1 (muy en desacuerdo) a 5 (muy de acuerdo) & Media & $\begin{array}{c}\text { Desv. } \\
\text { Est }\end{array}$ \\
\hline \multicolumn{4}{|l|}{ APRENDIZAJE } \\
\hline & El curso me ha parecido intelectualmente estimulante & 3,18 & 0,75 \\
\hline & He aprendido cosas que considero valiosas & 3,82 & 0,87 \\
\hline & Mi interés en la materia ha aumentado & 3,27 & 1,27 \\
\hline & He aprendido y comprendido los contenidos de este curso & 3,91 & 0,30 \\
\hline \multicolumn{4}{|l|}{ ENTUSIASMO } \\
\hline & Los profesores han mostrado entusiasmo & 3,36 & 0,92 \\
\hline & Los profesores han sido dinámicos y activos & 3,36 & 1,03 \\
\hline
\end{tabular}




\begin{tabular}{|c|c|}
\hline Los profesores consiguen que sus presentaciones resulten amenas & $2,91 \mid$ \\
\hline $\begin{array}{r}\text { Las explicaciones de los profesores eran claras } \\
\text { El material del curso estaba bien preparado } \\
\text { Los objetivos anunciados coincidieron con lo que se enseñó. He estado } \\
\text { orientado } \\
\text { La forma en que los profesores exponían la materia me ha hecho fácil tomar } \\
\text { apuntes }\end{array}$ & 2,73 \\
\hline \begin{tabular}{|} 
INTERACCIÓN CON EL GRUPO \\
Se animaba a los estudiantes a participar en las discusiones de clase \\
Se invitaba a los estudiantes a compartir sus conocimientos e ideas \\
Se animaba a los estudiantes a preguntar y se los daba respuestas satisfactorias \\
Se animaba a los estudiantes a expresar sus ideas y a cuestionar las expresadas \\
por los profesores
\end{tabular} & $\begin{array}{l}4,18 \\
4,27 \\
3,55\end{array}$ \\
\hline \begin{tabular}{|} 
ACTITUD PERSONAL \\
Los profesoreses se han mostrado accesibles en el trato individual con los \\
estudiantes \\
Los profesores me hacían sentir bien recibido cuando les pedía ayuda o consejo \\
Los profesores han mostrado interés sincero por todos los alumnos \\
Los profesores estaban disponibles para los estudiantes fuera de las horas de \\
clase
\end{tabular} & $\begin{array}{l}3,82 \\
4,00 \\
4,00\end{array}$ \\
\hline \begin{tabular}{|r} 
CONTENIDOS \\
Los profesores analizaron las implicaciones de planteamientos alternativos a las \\
teorías expuestas \\
Los profesores presentaron el fundamento de las ideas desarrolladas en clase \\
Los profesores presentaron puntos de vista diferentes a los suyos \\
Los profesores discutieron de forma adecuada los avances actuales en la \\
materia
\end{tabular} & $\begin{array}{l}3,18 \\
3,09 \\
3,45 \\
3,50\end{array}$ \\
\hline \begin{tabular}{|} 
EXÁMENES \\
$\begin{array}{r}\text { Los comentarios de los profesores sobre los exámenes y trabajos corregidos } \\
\text { fueron de gran ayuda }\end{array}$ \\
Los métodos de evaluación de este curso son equitativos y adecuados \\
Los contenidos de las evaluaciones se correspondían con los contenidos y el \\
énfasis de cada tema
\end{tabular} & $\begin{array}{l}2,73 \\
3,27 \\
3,45\end{array}$ \\
\hline $\begin{array}{r}\text { El método docente contribuye a mejorar la valoración y la comprensión de } 1 \\
\text { materi }\end{array}$ & 3,55 \\
\hline
\end{tabular}




\begin{tabular}{|c|c|c|}
\hline CARGA DE TRABAJO Y DIFICULTAD & & \\
\hline Este curso comparado con otros, ha sido... & 3,00 & 0,77 \\
\hline La carga de trabajo de este curso comparado con otros, ha sido... & 4,27 & 0,79 \\
\hline El ritmo del curso ha sido... & 3,55 & 0,82 \\
\hline En media, las horas por semana de trabajo fuera de clase han sido... & 3,09 & 1,14 \\
\hline VISIÓN GENERAL & & \\
\hline Este curso es mejor que la mayoría de los cursos de esta Universidad & & \\
\hline Estos profesores son mejores que la mayoría de los que he tenido en esta & & \\
\hline Universidad & 3,18 & 0,98 \\
\hline OTRAS OPINIONES SOBRE LA MATERIA Y EL CURSO & & \\
\hline Tu nivel de interés por la materia antes de hacer este curso era & 3,82 & 1,17 \\
\hline La calificación final que esperas obtener en este curso es.. & 3,73 & 0,47 \\
\hline
\end{tabular}

Desde el punto de vista docente, de asimilación de objetivos, los dos profesores valoramos muy positivamente la asignatura, que ha sido seguida con éxito por 21 de los 22 alumnos matriculados, manifestando su progreso en las habilidades y capacidades requeridas. En concreto, el nivel de análisis y su auto-confianza para sugerir mejoras a los artículos científicos previamente publicados ha evolucionado espectacularmente desde la primera a la tercera evaluación continuada. Lo mismo puede decirse de la calidad de sus exposiciones orales y escritas a partir de las presentaciones de los tres ejercicios cooperativos y de las prácticas. Su necesidad de ser críticos con el trabajo de sus compañeros, con un nivel de exigencia usualmente superior al que teníamos los profesores, ha sido un acicate para mejorar el nivel de sus propias presentaciones.

Sin embargo, la asignatura no ha sido percibida por los alumnos de forma tan satisfactoria como por los profesores. El uso de la lengua inglesa y el empleo de ejemplos médicos reales con terminología específica experimentaron una cierta resistencia inicial que finalmente fue superada. Por iniciativa de los profesores, un alumno recogió de forma anónima la encuesta SEEQ (Marsh y Roche, 1997), pero sólo fue contestada por 11 de los 21 alumnos. Los resultados cuantitativos, relativamente positivos, se reflejan en el cuadro 5.

Algunos de los comentarios recogidos en la parte abierta de la encuesta (Cuadro 6) hacen referencias a disfuncionalidades que asumimos como aceptables en la primera edición de una asignatura tan diferenciada y que pueden ser fácilmente corregibles en una nueva edición. Otras respuestas con menor puntuación se explican porque algunas preguntas están pensadas para una asignatura de metodología tradicional. Sin embargo, una interpretación conjunta de ambas evaluaciones obliga a reflexionar sobre las expectativas de los alumnos en un curso universitario y los roles de profesores y alumnos, con quejas muy explícitas sobre el nivel de responsabilidad que deben asumir todos los implicados.

Los dos profesores han percibido mayores cargas de trabajo por la necesidad de preparar con adelanto todos los materiales de trabajo y por la presión de los alumnos para recibir una retroalimentación individualizada. En cambio, la ya comentada implicación del alumno en la evaluación de los ejercicios de sus compañeros ha permitido disminuir progresivamente la presión al profesorado.

\section{Conclusiones}

La primera consideración debe ser que es posible hacer una docencia alternativa dentro de nuestra estructura universitaria. Aunque los alumnos ofrecen cierta resistencia al cambio, la valoración global de la primera edición de esta versión semi-presencial de la asignatura encaja con la media de las asignaturas de su entorno.

Cuadro 6: evaluación cualitativa del curso.

Por favor, indica cuáles son las características de este profesor/curso que te han ayudado más en el proceso

de aprendizaje de este curso. 
La veracidad de todos los ejemplos.

El carácter práctico de la asignatura y la participación activa del alumno.

Lo que más me ha ayudado a comprender el curso han sido las prácticas, porque era cuando realmente entendías punto por punto los criterios de cada lista y te surgían las dudas.

Experiencia y los conocimientos prácticos sobre la materia.

El hecho de hacer debates y hacer clases abiertas.

Libertad de horario, bueno para encontrar el mejor momento y el tiempo que se le quiere dedicar.

Por favor, indica cuáles son las características de este profesor/curso que se tendrían que mejorar de forma prioritaria (especialmente, aspectos no considerados a las preguntas anteriores).

La planificación.

La falta de correcciones de los ejercicios de e-status, la necesidad de enfocar los temas antes de hacer los resúmenes en grupo.

Personalmente las clases eran muy aburridas, está claro que no se marcó en un principio como tenían que ser las presentaciones, o sea, más dinámicas y con transparencias bien resumidas y claras. Creo que los profesores hablaban poco en las clases, me hubiese gustado mucho más que hubiesen puesto ejemplos de situaciones reales.

Más explicaciones en clase sobre los temas trabajados.

Demasiada carga de trabajo, lo que hace la asignatura un poco pesada. Además, sólo son 6 créditos por tanto trabajo y la mayoría de alumnos trabajan y hay más asignaturas.

Por favor, utiliza el espacio adicional para clarificar cualquiera de tus respuestas o para añadir cualquier comentario complementario.

En este curso se han equiparado las horas que no se hacían en clase con las de trabajo fuera de clase. Para mí es un error ya que el esfuerzo que te exigen unas y otros es diferente. A mí personalmente me resulta mucho más descansado asistir una hora a clase que estudiar una hora por mi cuenta.

La asignatura me ha gustado mucho a pesar de que llevaba mucho trabajo, pero creo que el sistema de clases no es el óptimo. Personalmente, me hubiese gustado una clase con más preguntas, más dudas entre nosotros (siempre éramos las mismas las que preguntábamos), y que entre profesores y alumnos se mantuviesen más coloquios. El hecho de que cada grupo haga su presentación lo encontré aburrido.

Los alumnos que no disponen de internet el fin de semana tienen menos tiempo para la entrega de problemas con e-status, además el programa falla mucho y la calificación que se obtiene de estos ejercicios encuentro que es completamente aleatoria.

La segunda consideración debe ser la alta satisfacción de los profesores en cuanto al desarrollo de las habilidades de los alumnos para trabajar en grupo, hacer presentaciones orales y auto-aprender de manera autónoma. Especialmente espectacular ha sido el desarrollo de su sentido crítico y su capacidad para detectar, con la ayuda de las directrices internacionales, los puntos débiles de un original científico y sugerir mejoras en su presentación. A este nivel, las directrices de consenso internacional han sido un soporte muy eficaz. Aunque los profesores temíamos que el uso de listas de comprobación podía convertirse en ejercicio mecánico, desprovisto de contenido, la realidad ha ido en sentido contrario, ya que han permitido aflorar los puntos más conflictivos al mismo tiempo que aclaraban los más esenciales. Para favorecer la discusión y el desarrollo de la capacidad crítica, los dos profesores hemos evitado actitudes dogmáticas, si bien hemos resaltado que estandarizar la presentación de resultados aumenta su legibilidad y facilita su repercusión posterior.

En resumen, las dos primeras consideraciones apoyan la evolución marcada por la declaración de Bolonia, si bien el comentario final debe recordar que el camino no es fácil, debido a las resistencias al cambio por parte de los implicados. Debemos adecuar las cargas de trabajo de alumnos y profesores. Conviene, en especial, informar al alumno de las características del curso para que pueda iniciarlo con plena conciencia del trabajo que deberá realizar. Dado que la encuesta no ha sido contestada de forma mayoritaria, será interesante ver la 
evolución de la demanda de esta asignatura optativa.

\section{Agradecimientos}

Al jefe de estudios de la Licenciatura en Ciencias y Técnicas Estadísticas de la Facultad de Matemáticas y Estadística, Dr. Javier Heredia, por sus consejos y apoyo durante todo este proceso. Al estudiante Francesc Miras por su soporte en la elaboración de la encuesta y sugerencias durante el curso. Al comité editorial de Medicina Clínica por facilitar la incorporación de estadísticos en su proceso de revisión.

\section{Referencias}

ALTMAN D. y GOODMAN, S. (1994) Transfer of technology from statistical journals to the biomedical literature: past trends and future predictions. JAMA;272:129-32

ARNAU, C.; COBO, E. et al. (2003) Efecto de la revisión estadística en la calidad de los manuscritos en Medicina Clínica. Med Clin (Barcelona); 121:694-4.

BERGER, J. (2003) Could Fisher, Jeffreys and Newman have agreed on testing? Statistical Science 2003; 18:132.

BOSSUYT, P. et al. (2003). The STARD Statement for Reporting Studies of Diagnostic Accuracy: Explanation and Elaboration. Clinical Chemistry; 49:17-18.

FERNÁNDEZ, E. (2005) Estudios epidemiológicos (STROBE). Medicina Clínica. Vol. 125. 43-48

GONZÁLEZ, J.A. et al. (2006). Desarrollo y Aplicación de Nuevas Tecnologías para la Formación Universitaria. COMPLETAR REFERENCIA.

GORDON, C; SMITH, S. y PELL, J.P. (2003) Parachute use to prevent death and major trauma related to gravitational challenge: systematic review of randomised controlled trials. $B M J ; 327 ; 1459-1461$

HEREDIA, J. (2005). Memòria del Pla Pilot del Grau de Estadística. FME, UPC.

http://www-fme.upc.es/docs-fme/050520_Objectius_competencies_GE.pdf

HUBBARD, R. y BAYARRI, M.J. (2003) Confusion over measures of evidence (P's) versus errors (a's) in classical statistical testing. The American Statistician; 57: 171-82.

MARSH, H.W. y ROCHE, L.A. (1997). Making Students' Evaluations of Teaching Effectiveness Effective: Critical Issues of Validity, Bias, and Utility. American Psychologist 52:1187-1197.

MINISTERIO DE EDUCACIÓN Y CIENCIA (1999). Espacio Europeo de Educación Superior. http://wwwn.mec.es/univ/jsp/plantilla.jsp?id=3501

MINISTROS DE EDUCACIÓN EUROPEOS (1999). El espacio europeo de la enseñanza superior. Declaración conjunta de los ministros europeos de educación reunidos en Bolonia el 19 de junio de 1999.

http://www.aneca.es/modal_eval/docs/declaracion_bolonia.pdf

MOHER D. (1998) CONSORT: an evolving tool to help improve the quality of reports of randomized controlled trials. Consolidated Standards of Reporting Trials. JAMA; 279:1489-91.

MOHER, D et al. (1999) Improving the quality of reports of meta-analyses of randomised controlled trials: the QUOROM statement. Lancet; 354: 1896-900.

POPPER, K. (1967) El desarrollo del conocimiento científico. Conjeturas y refutaciones. Ed. Paidos, Buenos Aires.

VON ELM, E. y EGGER, M. (2004) The scandal of poor epidemiological research. BMJ; 329:868-869. 
(C) Ediciones Universidad de Salamanca 